\title{
Algebraic Observability Analysis of Electrical Network with Symbolic Computation: Application on MYRTE Electrical Power Plant
}

\author{
Olivier Wailly*, Nicolas Héraud*, Eric J. R. Sambatra** \\ * SPE-CNRS UMR 6134, Université de Corse, BP 52 - Quartier Grossetti \\ 20250 Corte, France (mail : wailly@univ-corse.fr, heraud@univ-corse.fr). \\ **Institut Supérieur de Technologie, 201 - Antsiranana, BP 509 \\ Madagascar (mail: ericsambatra@yahoo.fr)
}

\begin{abstract}
We present a symbolic algebraic electrical system analysis method. A single-phase model is applied to a renewable electrical power plant. Once the system relations established, algorithms are applied, calculating the Groëbner bases for determining the system observability. Parallel programming is used in order to manage the number of relations and to decrease the time computing. A cost sensor placement is finally proposed to improve the observability.
\end{abstract}

Keywords: electrical network, grid, diagnosis, observability, sensor design, symbolic computation, algorithm

\section{Introduction}

In electrical power distribution, the network efficiency is a priority. Especially, in renewable sources, knowing the effective losses in particular conditions can be an argument to prove the importance of an electric power supply installation. Furthermore, looking for grid security, current researches are heading for smart grid, where grid security depends of the security of every dispatched source (Sioshansi 2011).

To have a good security level in electrical power plant, like in any plant, it is a necessity to have perfect system-state knowledge. Thus, it is required to make system state estimation.

Having regard to this information, the state estimation quality depends on sensor position and reliability and also of exhaustiveness knowledge of relations and their redundancy.

Two main ways exist in system design to elaborate a system relation complete catalog, algebraic (Ragot \& al. 1991, Frisk \& al. 2009, Hurtgen \& Maun 2010, Vitus \& al. 2012) or graph's theory (Krumpholz \& al. 1980, Chen 1990, Wu 2013). For a few years, we developed an algebraic method, based on symbolic computation (Héraud 2005), permitting to have a complete catalog of the system relations and to know redundant relations of existing layout. Furthermore, we developed algorithms that help engineers to make sensor design with multi criterion cost. Recently, we presented a new algorithm organization using parallel computing in order to manage large scale plants (Wailly \& al. 2013).

Our laboratory has at one's disposal a new photovoltaic (PV) electrical supply plant. This fulfills controlled electrical providing function. One of final goals is to be able to provide electrical power with controlled pattern as demanded by electrical providing society. This site contains PV panels, an electrolyzer, hydrogen and oxygen storage and a fuel cell. Obviously, the place contains a substantial electrical grid where security is of vital importance. In order to make the electrical supervision and eventual sensor upgrade, we present here the MYRTE electrical grid analysis with our algebraic method to know the exact available relations and to consider sensor network evolution.

\section{The method}

\subsection{Presentation}

For an existing configuration, it is important to isolate the observable part of the system. Figure 1 shows how must be made the analysis.

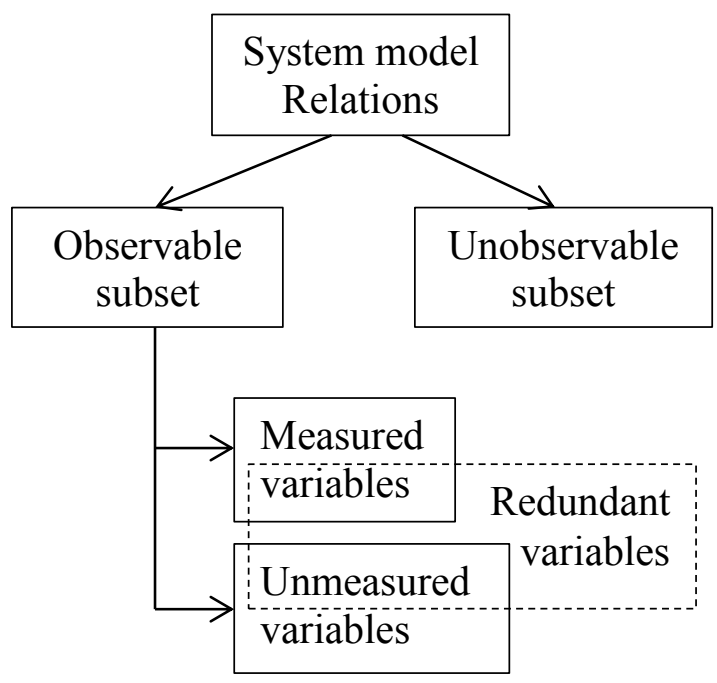


Figure 1 Observability analysis

Here, we use the following notations:

$F$ : System model with $f$ polynomial relations $V=\left\{x_{1}, x_{2} \ldots \quad, \quad\right.$ st of $v$ variables involved in $F$

$k=\left(\begin{array}{c}v \\ v-f\end{array}\right)$ : Number of set combinations

(1) System Model

\subsection{Definitions}

The method developed here is an application of Groëbner bases theory. So, we must first put down some mathematical definitions taken from Xia S. and Xia G. 2009.

Definition 1 Let $I$ be a nonzero ideal of a ring $A, G=\{g l, \ldots$, $g s\}$ be a nonzero finite set of polynomials. The set $G$ is called a Gröebner basis of the ideal $I$, if and only if for each polynomial $f$ in $I$, there exists $j, 1 \leq j \leq s$, such that $\operatorname{lp}\left(g_{j}\right) \mid \operatorname{lp}(f)$, where $\operatorname{lp}(f)$ is the leader product of power of $f$.

Definition 2 For polynomials $f, g, h$ in a ring $A, g \neq 0, f$ is called one-step reduce to $h$ by module $g$, denoted by $f \stackrel{g}{\rightarrow} h$, if and only if $l p(g)$ is a factor of nonzero monomial expression $X$ of $f$, and $h=f-\frac{X}{l t(g)} g$, where $\operatorname{lt}(\mathrm{g})$ denotes the leader of g.

Definition 3 Let $f, f_{l}, \ldots, f_{s}$ and $f_{j} \neq 0(1 \leq j \leq s)$, set $F=\left\{f_{l}, \ldots\right.$, $\left.f_{s}\right\}, f$ is called reduce to $h$ about module $F$, denoted by $f \stackrel{F}{\rightarrow}+{ }_{+} h$, if and only if $f \stackrel{f_{i 1}}{\rightarrow} h_{1} \stackrel{f_{i 2}}{\rightarrow} h_{2} \rightarrow \cdots \stackrel{f_{i t}}{\rightarrow} h_{t}=\mathrm{h}$ where $(j=1, \ldots, t)$.

Theorem $\boldsymbol{A}$ (Buchberger 2006) Let $I$ be a nonzero ideal of a ring $A=\left[x_{1}, \ldots, x_{n}\right], G=\left\{f_{l}, \ldots, f_{t}\right\} \subseteq \Lambda\{0\}$, then the followings are equivalent:

(a) $G$ is a Gröbner basis of $I$

(b) $f \in I$ if and only if $f \stackrel{G}{\rightarrow}++0$.

Kobayashi 1988, Yokoyama 1990 and Giusti 2001 have used this theory in order to develop algorithms for solving polynomial systems.

Our algorithms use GB to present the system in a triangular form (Wailly \& al. 2007). We showed that this form of the system permit to isolate deduced variables from a measured variable vector.

\subsection{Algorithms}

With symbolic computation, by means of Groëbner bases (GB), the first needed thing is to determine the necessary minimal number of variables for system observability. With the precedent notation defined in (1), this number is $(v-f)$.
If the number of sensors is just equal to $(v-f)$, GB can be calculated. Then, polynomials obtained are watched to know which variables are either or not deductibles. The GB gives a triangular form as far as possible. If the number of sensors is greater than the minimum, it is not so direct. To isolate observable islands in the sensor set, we need to know every minimal combination sets and to search by combinatorial operations the island (Wailly O. \& al. 2007).

Thus, in sensor network design, the method consists to construct a minimum variable observable set data base of the system first. Afterwards, knowing observability or isolating observable islands will mean to make combinatorial combinations between data base and sensor solutions.

To sum up, we present now an algorithm for this first part (Algorithm 1) according to (1) definition:

1. Define the system

2. Calculate every set combinations with $v-f$ variables

3. Isolate the observable combinations (using GB) and put it away in data base

4. Isolate the system observable and redundant part with measurements (using combinatorial operations between measurement set and data base)

This algorithm has only a few operations but it can be time consuming. If combinatorial operations are quite fast in symbolic computation, the exponential growing of sets brings some difficulties. GB algorithms are them by nature time consuming. So, we develop a non-recursive algorithm so as to overcome this inconvenient and adapt our algorithm with parallel computing in order to manage large scale systems.

\subsection{Cost criteria algorithm}

When a database has been constructed with all observable possibility the cost criteria can be added. For each unit cost a weight can be assigned. Then a matrix can be developed in order to establish which combination is the most cost efficient. This method is flexible as each 'weight' can be changed depending on the drivers (Wailly \& al. 2005).

We can present the following algorithm to explain the criteria method (Algorithm 2):

1. Add weight for each cost criteria

2. Present results in such a way to select preferred one

We applied this algorithm up to 3 criteria, introducing cost, technical and accuracy.

These operations are less time consuming than the preparation of the solution database. For the systems tested in evaluation, it was showed that it took less than a few seconds for medium size plant, which is perfectly adapted for common cases.

\subsection{About computing time}


The computing time can be a limitation of symbolic computation use in lot of applications. In Wailly 2013, we evaluated the computing time problem and we proposed an algorithm that permits to use GB for extended system. We particularly showed that the empiric laws of grid computing are close to the results we found.

\section{MYRTE plant scheme}

\subsection{MYRTE description}

The MYRTE platform was inaugurated in 2011 (Darras 2012, Myrte 2012) and is situated at University of Corsica, Route des Sanguinaires, F-20000 Ajaccio, France (Figure 2). It is one of the PEPITE (Study and experimentation of intermittent energy management using electrochemical technologies) project applications included in the PAN-H (national action plan on the hydrogen and the fuel cells) program of the ANR (French research national agency), under the reference ANR-07-PANH-012. This platform comes from a partnership between the University of Corsica, the HELION Company subsidiary from AREVA and the CEA (atomic energy and alternative energies commission, French government-funded technological research organization) and the CNRS (French National Center for Scientific Research). Studies are entrusted to UMR CNRS SPE 6134, University of Corsica.

The MYRTE platform is a technological platform dedicated to $\mathrm{PV} / \mathrm{H} 2$ coupling study. It was inaugurated on Monday, January 9th, 2012. The main objective is to test the hydrogen technology in real situation, and to develop optimal operating strategies between a PV array (solar panels and electric inverters) and a $\mathrm{H} 2$ chain (electrolyzer; $\mathrm{H} 2, \mathrm{O} 2$ and $\mathrm{H} 2 \mathrm{O}$ tanks; and fuel cells).

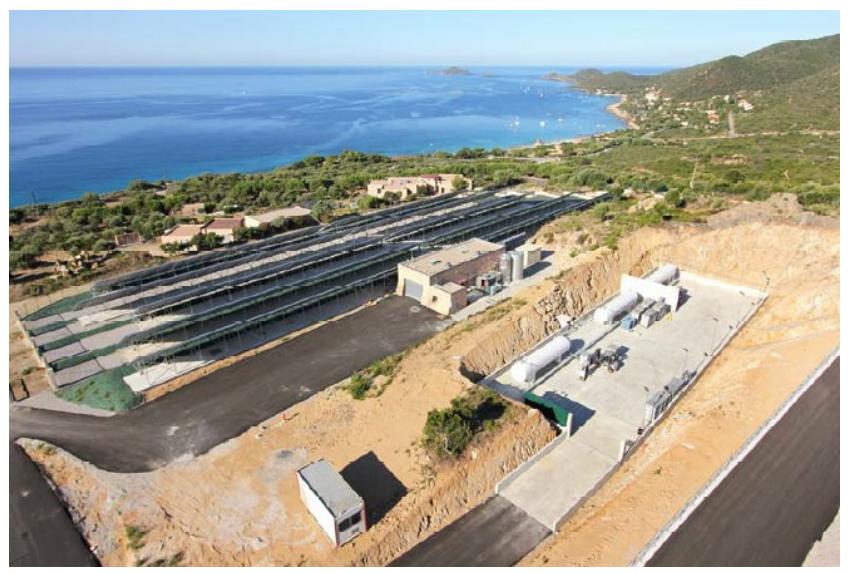

Figure 2 MYRTE overview

The MYRTE platform objectives are to study controlled and intelligent providing of the PV electrical production to electrical supplier. Different production modes have been studied or are under study.

The main power elements are:

- A 2240-photovoltaic-modules photovoltaic array of $560 \mathrm{kWp}$
- A fuel cell with nominal power at $210 \mathrm{~kW}$ delivering electricity through a DC/AC inverter

- An $200 \mathrm{~kW}$ electrolyzer using AC/DC inverter

- Four H2 tanks and two O2 tanks for gas storage

- A thermal management system of a capacity of 800 $\mathrm{kWh} /$ day

- An $800 \mathrm{kVA}$ transformer providing high voltage to electrical distribution grid

Figure 3 gives a SCADA view of electrical power management. The main power parts detailed above can be spotted.

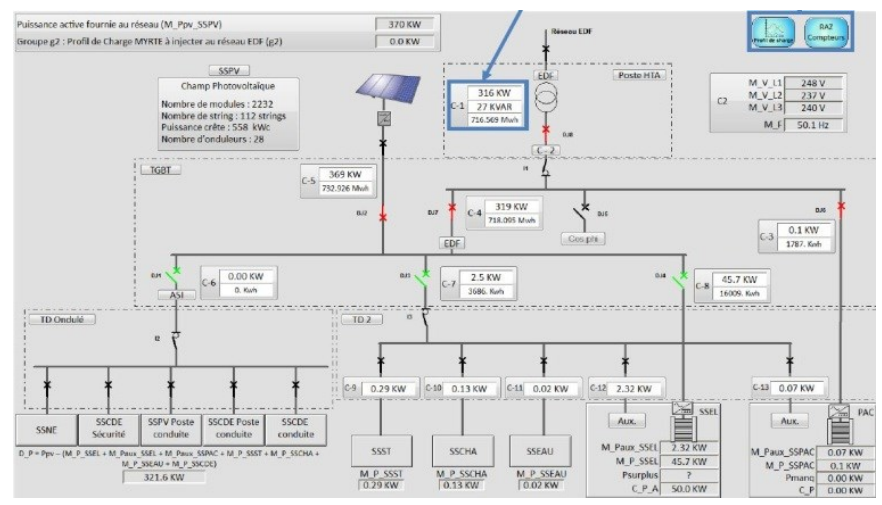

Figure 3 SCADA view of electrical power plant

In the following study, we will use a single-phase approximation. From SCADA view, we can obtain the scheme presented at figure 4. On the figure, we find distribution transformer, the three DC/AC converters. Secondary consumptions have been concentrated in one branch and virtually represented as a load.

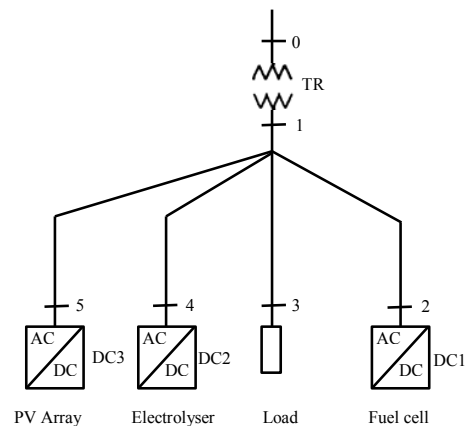

Figure 4 Scheme of the electrical power plant

\subsection{Single phase component model}

Model chosen are classical ones.

The converters (DC1 to DC3) are represented by a terminal bus $\mathrm{H}$ (external) and an internal bus I (internal), according to figure 5. Relations are expressed in (2).

The transmission line model is represented at figure 6 . This transmission line is considered as symmetrical $\left(Y_{A}=Y_{B}\right)$ and the relations are presented in (3). 


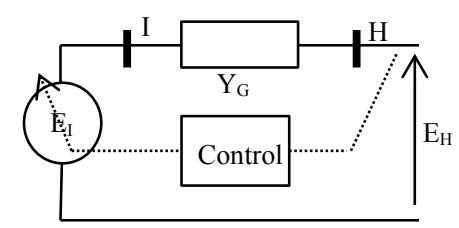

Figure 5 Converter model

$$
\left(\begin{array}{c}
i_{I} \\
i_{H}
\end{array}\right)=\left(\begin{array}{cc}
Y_{G} & -Y_{G} \\
-Y_{G} & Y_{G}
\end{array}\right)\left(\begin{array}{l}
e_{I} \\
e_{H}
\end{array}\right)
$$

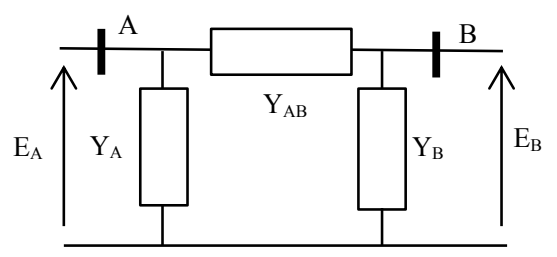

Figure 6 Transmission line

$$
\left(\begin{array}{l}
i_{A} \\
i_{B}
\end{array}\right)=\left(\begin{array}{cc}
Y_{A B}+Y_{A} & -Y_{A B} \\
-Y_{A B} & Y_{A B}+Y_{A}
\end{array}\right)\left(\begin{array}{l}
e_{A} \\
e_{B}
\end{array}\right)
$$

The transformer model is represented at figure 7. It is considered as symmetrical and the relations obtained are exposed in (4).

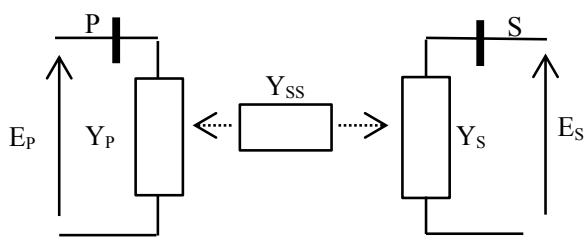

Figure 7 Transformer model

$$
\left(\begin{array}{l}
i_{P} \\
i_{S}
\end{array}\right)=\left(\begin{array}{ll}
Y_{P P} & Y_{P S} \\
Y_{P S} & Y_{P P}
\end{array}\right)\left(\begin{array}{l}
e_{P} \\
e_{S}
\end{array}\right)
$$

The load model chosen is presented at figure 8 and its relation expressed in (5).

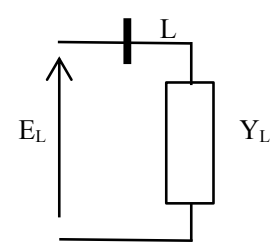

Figure 8 Load model

$$
i_{A}=Y_{A} \cdot e_{A}
$$

The relations coming from electrical laws with the models above, applied to scheme presented at figure 4, gives a system of 17 relations with 32 variables (Appendix A). It means that the minimum measurement set must have 15 elements. So, this subset combinations of 15 elements are the number of 565722720 .

\section{Results}

First, we have to take into account the measurement vector (appendix B-1). With these, we obtain by means of GB the vector given in (appendix B-2), which correspond to deduced variables. The first interest is that the deduction relations are explicit from GB and given in (appendix B-3).

But the system is not observable. It needs three measurements more. Applying Algorithm 1, we found that there are 84 candidates of 3-tuples variables from the set of variables minus the set of measurements. Examining these sets, we found 27 sets making the system observable. They are presented in appendix $\mathrm{C}-1$. Every deduction relation are directly gathered but not presented here for space reason.

The cost algorithm, algorithm 2, is applied on the 27 observable sets, using the cost vector in appendix C-2. This cost vector is made with technical cost of each measurement. The best solution can be determined from the result presented in appendix C-3.

\section{Conclusions}

We have here presented a new algebraic method applied to electrical network. This application was possible due to recent development we made on our algorithms and using parallel computing. We have chosen single-phase models. This model gives a first approach of the analysis. Anyway, it is commonly admitted that single-phase model is not often sufficient for small electrical network. It seems so necessary to pursue the present work and to improve the modeling to three-phase models.

\section{References}

Buchberger B. (2006) Bruno Buchberger's PhD thesis 1965: An algorithm for finding the basis elements of the residue class ring of a zero dimensional polynomial ideal. Journal of Symbolic Computation 41, 475-511.

Chen RL. (1990) A fast integer algorithm for observability analysis using network topology. IEEE Trans Power Syst 1990;5:1001-9.

Darras C., Muselli M., Poggi P., Voyant C., Hoguet J.-C., Montignac F. (2012) PV output power fluctuations smoothing: The MYRTE platform experience, International Journal of Hydrogen Energy 37 p.140154025.

Frisk E., Krysander M., Åslund J. (2009), Sensor placement for fault isolation in linear differential-algebraic systems, Automatica, 45, 364-371.

Giusti M., Lecerf G., Salvy B. (2001) A Groëbner Free Alternative for Polynomial System Solving, Journal of complexity 17, 154-211.

Héraud N., Wailly O., Bennouna O., Poggi P. (2005), On the topological observability of an electrical system, IEEE, Eleventh International Conference on Electrical Machines, Drives and Power Systems, Bulgaria. 
Hurtgen M, Maun JC. (2010) Optimal PMU placement using iterated local search. International Journal Electrical Power Energy Syst 2010;32(8):857-60.

Kobatashi H., Fujise T., Furkawa A. (1988) Solving Systems of Algebraic Equations by a General Elimination Method, Journal of Symbolic Computation 5, 303-320.

Krumpholz GR, Clements KA, Davis PW. (1980) Power system observability: a practical algorithm using network topology. IEEE Trans Power App Sys;PAS-99 (4):1534-42.

Myrte 2012: Available information via the link: http://myrte.univ-corse.fr/Inauguration-de-laplateforme-MYRTE- al.html.

Ragot J., Maquin D., Bloch G., Gomolka W. (1991), Observability and variables classification in bilinear processes, Journal $A$.

Sioshansi F. (2011) So What's So Smart about the Smart Grid? December, The Electricity Journal, Vol. 24, Issue 10 1040-6190.

Vitus M. P., Zhangb W., Abate A., Hud J., Tomlin C. J. (2012). On efficient sensor scheduling for linear dynamical systems. Automatica, 48, 2482-2493.

Wailly O., N. Héraud. (2005). Cost-optimal design of reliable sensor networks extended to multi-linear system. Computers and Chemical Engineering, Volume 29, Issue 5, P. 1083-1087.

Wailly O., Héraud N., Malassè O. (2007). Design of Instrumentation in Process Plants using Groëbner Bases. Computers and Chemical Engineering, Volume 32, Issue 10, Pages 2179-2188.

Wailly O., Héraud N., Sambatra E. (2013). Evaluating sensor placement on extended system with polynomial symbolic calculus. IFAC MIM'2013, Saint Petersburg, Russia, June 19-21.

Wu Z., Hsieh S.-J., Li J. (2013). Sensor deployment based on fuzzy graph considering heterogeneity and multipleobjectives to diagnose manufacturing system. Robotics and Computer-Integrated Manufacturing, 29, 192-208.

Xia S. and Xia G. (2009). An Application of Gröbner Bases. The Montana Mathematics Enthusiast, ISSN 1551-3440, Vol. 6, no.3, pp.381-394, Montana Council of Teachers of Mathematics \& Information Age Publishing.

Yokoyama K., NORO M., Takeshima T. (1992) Solutions of Systems of Algebraic Equations and Linear Maps on Residue Class Rings. Journal of Symbolic Computation 14, 399-417.

\section{Appendix A. System model}

$$
\begin{aligned}
& \left(\begin{array}{l}
I_{0} \\
I_{1}
\end{array}\right)=\left(\begin{array}{ll}
Y_{00} & Y_{01} \\
Y_{01} & Y_{00}
\end{array}\right)\left(\begin{array}{l}
E_{0} \\
E_{1}
\end{array}\right) \quad(\mathrm{TR})\left(\begin{array}{c}
I_{1 I} \\
I_{2}
\end{array}\right)=\left(\begin{array}{cc}
Y_{G 1} & -Y_{G 1} \\
-Y_{G 1} & Y_{G 1}
\end{array}\right)\left(\begin{array}{l}
E_{1 I} \\
E_{2}
\end{array}\right)(\mathrm{DC} 1) \\
& \left(\begin{array}{l}
I_{1} \\
I_{2}
\end{array}\right)=\left(\begin{array}{cc}
Y_{12}+Y_{2} & -Y_{12} \\
-Y_{12} & Y_{12}+Y_{2}
\end{array}\right)\left(\begin{array}{l}
E_{1} \\
E_{2}
\end{array}\right) \text { (1-2 line) } I_{3}=Y_{3} \cdot E_{3} \quad(\mathrm{Load}) \\
& \left(\begin{array}{l}
I_{1} \\
I_{3}
\end{array}\right)=\left(\begin{array}{cc}
Y_{13}+Y_{3} & -Y_{13} \\
-Y_{13} & Y_{13}+Y_{3}
\end{array}\right)\left(\begin{array}{l}
E_{1} \\
E_{3}
\end{array}\right) \text { (1-3 line) }\left(\begin{array}{c}
I_{2 I} \\
I_{4}
\end{array}\right)=\left(\begin{array}{cc}
Y_{G 2} & -Y_{G 2} \\
-Y_{G 2} & Y_{G 2}
\end{array}\right)\left(\begin{array}{c}
E_{2 I} \\
E_{4}
\end{array}\right) \text { (DC2) } \\
& \left(\begin{array}{l}
I_{1} \\
I_{4}
\end{array}\right)=\left(\begin{array}{cc}
Y_{14}+Y_{4} & -Y_{14} \\
-Y_{14} & Y_{14}+Y_{4}
\end{array}\right)\left(\begin{array}{l}
E_{1} \\
E_{4}
\end{array}\right) \text { (1-4 line) }\left(\begin{array}{c}
I_{3 I} \\
I_{5}
\end{array}\right)=\left(\begin{array}{cc}
Y_{G 3} & -Y_{G 3} \\
-Y_{G 3} & Y_{G 3}
\end{array}\right)\left(\begin{array}{c}
E_{3 I} \\
E_{5}
\end{array}\right) \text { (DC3) } \\
& \left(\begin{array}{l}
I_{1} \\
I_{5}
\end{array}\right)=\left(\begin{array}{cc}
Y_{15}+Y_{5} & -Y_{15} \\
-Y_{15} & Y_{15}+Y_{5}
\end{array}\right)\left(\begin{array}{l}
E_{1} \\
E_{5}
\end{array}\right) \text { (1-5 line) }
\end{aligned}
$$

\section{Appendix B. Observability study}

1- $\quad\{E 0, E 1, E 2, E 3, E 4, E 5,10,11,12,13,14,15\}$

2- $\quad\{Y 00, Y 01, Y 12, Y 13, Y 14, Y 15, Y 2, Y 3, Y 31, Y 4, Y 5\}$

3- $\quad\left(\mathrm{E} 1^{2}-\mathrm{E} 4 * \mathrm{E} 5\right) * \mathrm{Y} 5-\mathrm{E} 1 * \mathrm{I} 1-\mathrm{E} 1 * I 5+\mathrm{E} 4 * \mathrm{I} 1+\mathrm{E} 5 * \mathrm{I} 5, \quad(\mathrm{E} 1+\mathrm{E} 4) * \mathrm{Y} 4-\mathrm{I1}-\mathrm{I4}$, $\mathrm{E} 3 * \mathrm{Y3I}-\mathrm{I3}, \quad(\mathrm{E} 1+\mathrm{E3}) * \mathrm{Y} 3-\mathrm{I1}-\mathrm{I3}, \quad(\mathrm{E} 1+\mathrm{E} 2) * \mathrm{Y} 2-\mathrm{I1}-\mathrm{I2}, \quad\left(\mathrm{E} 1^{2}\right.$ $\mathrm{E} 4 * \mathrm{E} 5) * \mathrm{Y} 15+\mathrm{E} 1 * \mathrm{I5}-\mathrm{E} 4 * \mathrm{I} 1, \quad\left(\mathrm{E} 1^{2}-\mathrm{E} 4^{2}\right)^{*} \mathrm{Y} 14+\mathrm{E} 1 * I 4-\mathrm{E} 4 * \mathrm{I}, \quad\left(\mathrm{E} 1^{2}-\right.$ $\left.\mathrm{E}^{2}\right)^{*} \mathrm{Y} 13+\mathrm{E} 1 *|3-\mathrm{E} 3 *| 1, \quad\left(\mathrm{E}^{2}{ }^{2}-\mathrm{E}^{2}\right)^{*} \mathrm{Y} 12+\mathrm{E} 1 *|2-\mathrm{E} 2 *| 1, \quad\left(\mathrm{EO}^{2}-\right.$ $\left.\mathrm{E} 1^{2}\right) * \mathrm{Y} 01-\mathrm{E} 0 * \mid 1+10 * \mathrm{E} 1, \quad\left(\mathrm{EO}^{2}-\mathrm{E} 1^{2}\right) * \mathrm{Y} 00-\mathrm{E} 0 * 10+\mathrm{E} 1 * \mathrm{I} 1$, $2 * E 5 * Y G 3+|3|+|5, \quad 2 * E 4 * Y G 2+| 2|+| 4, \quad 2 * E 2 * Y G 1+|1|+\mid 2$, $\mathrm{E} 31 * \mathrm{YG} 3+\mathrm{E} 5 * \mathrm{YG} 3+15, \mathrm{E} 21^{*} \mathrm{YG} 2+\mathrm{E} 4 * \mathrm{YG} 2+14, \mathrm{E} 11^{*} \mathrm{YG} 1+\mathrm{E} 2 * \mathrm{YG} 1+12$

\section{Appendix C. Cost study}

1- $\{E 1|, E 2|, E 3 \mid\},\{E 1|, E 2|,|3|\},\{E 1|, E 2|, Y G 3\},\{E 1|, E 3|,|2|\},\{E 11$, $E 3 \mid, Y G 2\},\{E 1|| ,2|| 3 \mid\},,\{E 1|| 2 \mid,, Y G 3\},\{E 1|| 3 \mid,, Y G 2\},\{E 1 \mid, Y G 2$, $Y G 3\},\{E 21, E 31,|1|\},\{E 21, E 3 \mid, Y G 1\},\{E 21,|11| 3 \mid\},,\{E 2|| 1 \mid,, Y G 3\}$, $\{E 21,|3|, Y G 1\},\{E 2 \mid, Y G 1, Y G 3\},\{E 3 I,|1|,|2|\},\{E 3|| 1 \mid,, Y G 2\},\{E 3 \mid$, $121, Y G 1\},\{E 3 \mid, Y G 1, Y G 2\},\{|1|,|2|,|3|\},\{|1|,|2|, Y G 3\},\{|1|,|3|$, YG2\}, \{11|, YG2, YG3\}, \{|2|, I3I, YG1\}, \{|2I, YG1, YG3\}, \{|3|, YG1, YG2\}, \{YG1, YG2, YG3\}

2- CostVector=[1, 1, 1, 5, 5, 5, 10, 10, 10]; CostOrder=[E1I, E2I, E3I, I1I, I2I, I3I, YG1, YG2, YG3]

3- $[3,7,12,7,12,11,16,16,21,7,12,11,16,16,21,11,16,16,21$, $15,20,20,25,20,25,25,30]$ in order of appendix C-1 